\title{
Optimization of the Force Transmission Path on the High-thrust Hyper-static Strap-on Launch Vehicle
}

\author{
Yong Mei ${ }^{1, a}$ \\ ${ }^{1}$ College of Aerospace Science and Engineering, \\ National University of Defense Technology, \\ Changsha, China, 410073 \\ a meiyong1990@sina.com

$$
\text { Yongjun Lei }{ }^{1, \mathrm{~b}, *}
$$ \\ ${ }^{1}$ College of Aerospace Science and Engineering, \\ National University of Defense Technology, \\ Changsha, China, 410073 \\ b,*1eiyj108@nudt.edu.cn
}

\author{
Ningyu Liu ${ }^{2, \mathrm{c}}$ \\ ${ }^{2}$ College of Mechanical and Vehicle Engineering, \\ Hunan University, \\ Changsha, China, 410082 \\ c1ny2009@163.com \\ Shaowei Feng ${ }^{3, d}$ \\ ${ }^{3}$ Beijing Institute of Space System Engineering, \\ Beijing, China, 100076 \\ d fawwi@126.com
}

\begin{abstract}
The strap-on linkage equipment on the current launch vehicle is more difficult to satisfy the load design requirement of the new generation high-thrust strap-on launch vehicle. According to the research of the new linkage equipment technology, this paper studies the force transmission path optimization and designs one category of hyper-static force transmission path schemes. Through the analysis, an optimal scheme of the force transmission path is selected. A combinatorial optimization strategy of the force transmission path of high-thrust strap-on launch vehicle is designed based on the iSIGHT platform. Firstly, multi-island genetic algorithm is used to explore the region where the globally optimal solution lies and then the exact optimal solution is approached by sequential quadratic programming. The optimized force transmission path can substantially reduce the load of the strap-on linkage equipment and also satisfy the requirement of the attitude control system design.
\end{abstract}

Keywords-High-thrust strap-on launch vehicle; Hyper-static strap-on; Force transmission path; Combinational optimization strategy

\section{INTRODUCTION}

The strap-on linkage equipment is an important part of the launch vehicle which is used to transmit the thrust of the booster to the core stage and thus effectively improves the transport capacity. A reasonable structural design of this equipment is the guarantee of the booster even the whole launch vehicle's safety. Besides, strap-on linkage equipment is usually installed in groups to constitute the force transmission path of the booster's thrust. The reasonable design of the force transmission path is the basis for the type selection, structural design and strength check of the strap-on linkage equipment. The new generation high-thrust strap-on launch vehicle's booster has improved greatly compared with the thrust of the understudied and active-duty launch vehicles in our country (the thrust of CZ-5's single booster is 240T)[1].
The current strap-on linkage scheme cannot meet the working requirement of the new generation high-thrust launch vehicle and a new type of force transmission path is needed so as to ensure the reliable strap-on linkage of the new generation high-thrust boosters.

This essay firstly designs one category of new hyper-static strap-on configurations and selects the optimal one through the strap-on linkage structure's load analysis under various external load conditions. Moreover, this essay integrates MSC.Patran/ Nastran, Matlab into iSIGHT and uses multi-island genetic algorithm and sequential quadratic programming to find optimal strap-on linkage parameters in the optimization space.

\section{OVERVIEW OF STRAP-ON LINKAGE SCHEMES}

Taking the globally common statically-determinate strap-on launch vehicle as an example, the strap-on linkage equipment is divided into the main linkage structure and the auxiliary linkage structure according to different load-bearing directions. And the former is also called the axial load bearing structure, which transmits the thrust from the booster and is the main load-bearing equipment and restricts the booster's degree of freedom of translation. The latter is also called the transverse load bearing structure, which transmits the shear force, torsion, radial load and restricts the booster's degree of freedom of rotation. The main characteristics of this kind of load-bearing combining scheme is to get the axial load and transverse load which are transmitted to the core stage from the booster borne by different equipment and achieve more accurate load analysis, thereby simplifying the structural design of the equipment. 

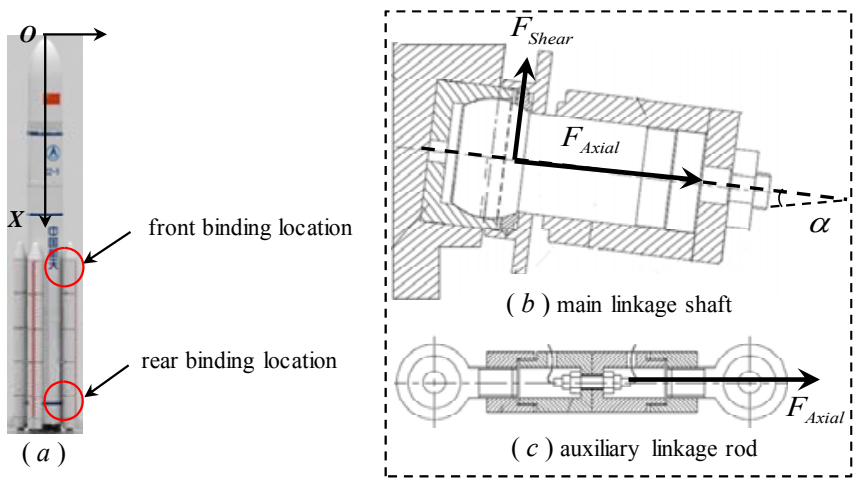

Fig.1 The linkage structure and installation position on the new generation high-thrust strap-on launch vehicle

The common main linkage scheme is showed like scheme A and B in Fig. 2 [3]. Ariane-V, CZ-5 take the front strap-on equipment as the main linkage structure, Titan-IIIC, Delta-IV and $\mathrm{CZ}-2 \mathrm{~F}$ take the front strap-on equipment as the main linkage structure. The auxiliary linkage structure of strap-on launch vehicle is used to transmit the radial load from the booster and restricts its lateral motion and rotation. As the load on this structure is usually small, the structure is relatively simple and designed as a multiple rod system. The common scheme is showed like scheme $\mathrm{C}$ and D in Fig. 2 [3].

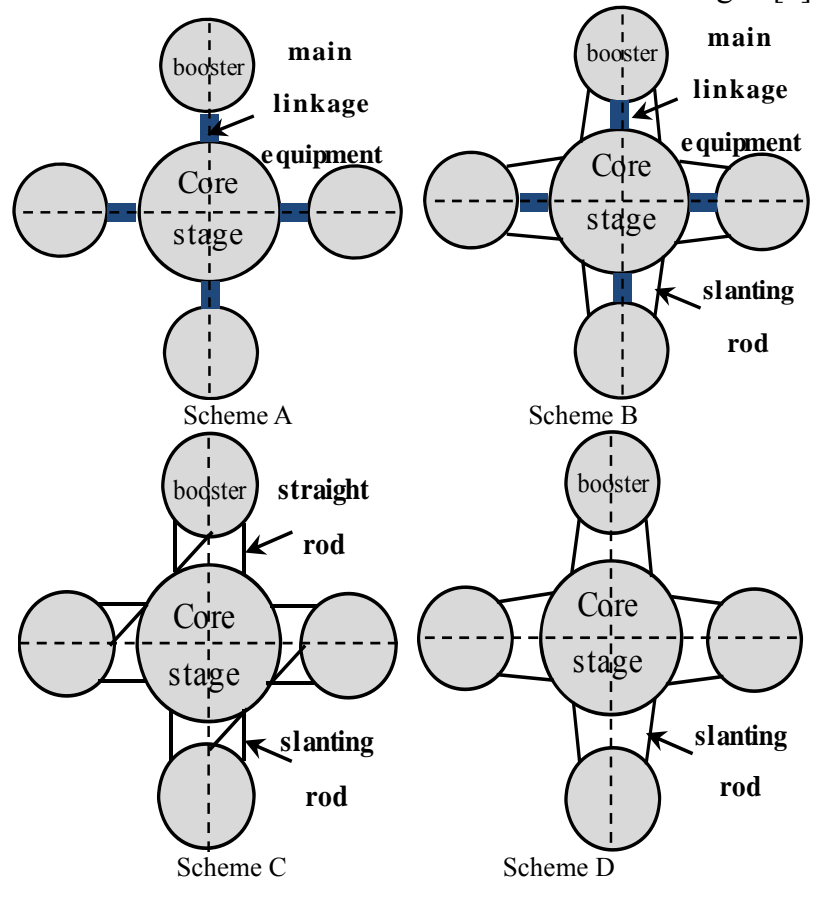

Fig.2 The common linkage scheme

\section{DESIGN OF THE FORCE TRANSMISSION PATH} OF HIGH-THRUST STRAP-ON LAUNCH VEHICLE

A. Load analysis on the linkage structure of the statically-determinate strap-on configuration
In the preliminary plan, the high-thrust launch vehicle (showed in Fig. 1(a) ) adopts the conventional scheme of statically-determinate strap-on configuration and its FEM model is showed in Fig. 4(b). The main strap-on structure employs scheme A see in Fig. 2, the auxiliary strap-on structure employs scheme $\mathrm{C}$ see in Fig. 2. When the front statically-determinate strap-on configuration is adopted, the whole launch vehicle employs the scheme of booster-fixed main support when being parked. When the rear statically-determinate strap-on configuration is adopted the whole launch vehicle employs the scheme of core-fixed main support. Table 1 shows the maximum load of the structure on the launch vehicle's strap-on linkage equipment under multiple external load conditions.

TABLE 1 MAXIMUM LOAD ON THE LINKAGE STRUCTURE OF THE STATICALLY-DETERMINATE STRAP-ON CONFIGURATION[KN]

\begin{tabular}{cccc}
\hline $\begin{array}{c}\text { force bearing } \\
\text { configuration }\end{array}$ & $\begin{array}{c}\text { auxiliary linkage } \\
\text { rod's } \\
\text { axial force }\end{array}$ & $\begin{array}{c}\text { main linkage } \\
\text { shaft's } \\
\text { axial force }\end{array}$ & $\begin{array}{c}\text { main linkage } \\
\text { shaft's } \\
\text { shear force }\end{array}$ \\
\hline $\begin{array}{c}\text { front strap-on } \\
\text { scheme } \\
\text { rear strap-on } \\
\text { scheme }\end{array}$ & 456.2 & -2123.3 & 4201.9 \\
\hline
\end{tabular}

For the main strap-on load-bearing part showed in Fig. 1(b), the ideal load-bearing form is the pressure and no tensile or shear ( the main index for the design of strap-on linkage is the pressure-bearing capacity, therefore its tension-bearing and shear-bearing capacity is relatively weak ). According to the computational results of rear strap-on scheme in table 1, we find that the main strap-on linkage shaft is in the state of tension. The further computation indicates the main shaft's state of tension will not vary with the change of the strap-on location (The strap-on linkage structure is in the state of tension when the launch vehicle is parked and in the state of compression when the launch vehicle is flying. The state's change of tension and compression will affect the perpendicularity of the whole launch vehicle [2]). Therefore, the front strap-on configuration is preferred when employing the statically-determinate strap-on configuration. The current large launch vehicles usually adopt the front strap-on configuration [5]. But under the front strap-on configuration, the main load-bearing structure bears much larger shear force than the maximum value of the current load-bearing structure's shear capacity. Therefore, it is needed to redesign the force transmission path of the strap-on linkage structure.

\section{B. Load analysis on the linkage structure of the hyper-static strap-on configuration}

The load on the strap-on launch vehicle is mainly borne by the main strap-on linkage structure. Therefore we try to install the main strap-on linkage equipment in the front and rear strap-on location on the new generation high-thrust launch vehicle and constitute the form of two-point main load bearing, which is analogous to Delta-IV. Two kinds of 
the main linkage equipment adopt scheme $\mathrm{A}$ and $\mathrm{B}$ showed in Fig. 2 respectively (The main strap-on equipment in scheme $\mathrm{B}$ is composed by a main strap-on structure and two auxiliary strap-on connecting rods. To simplify analysis, we use straight rod to replace the slanting rod). The FEM model of the high-thrust launch vehicle with new hyper-static strap-on configurations is showed in Fig. 3.

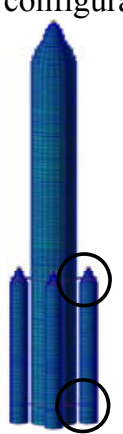

(a)

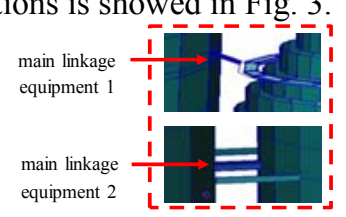

equipment

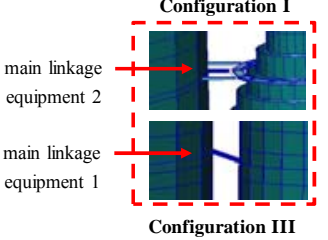

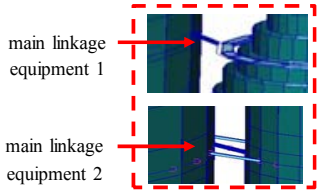

Configuration II

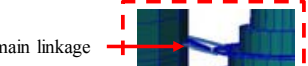

equipment 2

main linkage equipment 1

( $b$ ) objective, we study the influence of the coordinates of the front and rear strap-on location and the installation angle of the main linkage shaft (showed in Fig. 1(b) as $\alpha$ ) on the optimization objective. Meanwhile, the design of the force transmission path must take the requirement of the attitude control system's design into consideration, which means that the fundamental frequency of the whole launch vehicle should be smaller than the booster's local first-order frequency. Design variables' selection range is listed below:

1) The front strap-on location is located in the front cabinet of the booster's oxygen tank, the range of its coordinate is [61.994, 62.994], the length of the starp-on linkage part is $1 \mathrm{~m}$;

2) The rear strap-on location is located in the rear transitional part, the range of its coordinate is [92.722, 93.369], the length of the starp-on linkage part is $0.647 \mathrm{~m}$;

3) The range of the installation angle of the main strap-on shaft is $\left[11^{\circ}, 45^{\circ}\right]$.

The mathematical description of the multi-objective optimization problem for the strap-on force transmission path is

$$
\left\{\begin{array}{l}
\min \left(\mathrm{F}_{\text {Axial }}, \mathrm{F}_{\text {Shear }}, \mathrm{F}_{\text {Crod }}\right)^{\mathrm{T}} \\
\text { s.t. } \mathrm{g}_{\mathrm{i}}\left(\mathrm{x}_{\text {front }}, \mathrm{x}_{\text {behind }}, \alpha, \text { Freq }\right) \geq 0 \\
\text { Freq }_{\text {Horizontal }} \leq \text { Freq }_{\text {Booster }} \\
61.994 \leq \mathrm{x}_{\text {front }} \leq 62.994 \\
92.722 \leq \mathrm{x}_{\text {behind }} \leq 93.369 \\
11^{\mathrm{o}} \leq \alpha \leq 45^{\circ}
\end{array}\right.
$$

Objective function: $F_{\text {Axial }}$ (the main linkage shaft's maximum axial force), $F_{\text {Shear }}$ (the main linkage shaft's maximum shear force), $\mathrm{F}_{\text {Crod }}$ (the auxiliary linkage rod's maximum axial force);

Constraint function and design variables: $\mathrm{x}_{\text {front }}$ (the front strap-on location's coordinate), $\mathrm{x}_{\text {behind }}$ (the rear strap-on location's coordinate); $\alpha$ (the main strap-on shaft's inatallation angle); Freq is the launch vehicle's natural frequency, Freq Horizontal $_{\text {is }}$ the fundamental frequency of the transverse vibration on the launch vehicle, Freq booster $_{\text {is }}$ the first-order frequency of the booster.

This essay selects the global algorithm: Multi-island Genetic Algorithm [6] (MIGA) and local algorithm: Sequential Quadratic Programming [7] (SQP) to construct combinatory optimization strategy to conduct the optimization design of the force transmission path. This method can avoid potential large solving time caused by global algorithm and local optimal solution caused by local algorithm. The global algorithm can provide an excellent interval where the optimal solution lies in after several times of iteration. The local algorithm continues to explore in the excellent interval, making the algorithm converges to the optimal solution faster [8]. This method improves the optimization efficiency and the optimization result is rather 
accurate.

iSIGHT is a multi-disciplinary design optimization software integrating industrial optimization design and automation analysis which is promoted by Engineous Software Inc in Americas. Nowadays, iSIGHT has been widely used in aerospace, automotive, electronic, mechanical, chemical and other industries.

A complete iSIGHT optimization simulation procedure needs to achieve three basic tasks, which are modifying the model, submitting the analysis and extracting the computational results. This essay achieves the solution of high-thrust launch vehicle's optimization model and design variables' updated iteration by integrating MSC.Patran/ Nastran and Matlab in iSIGHT. The FEM model's pretreatment, backstage solving and the result's aftertreatment can be automatically achieved by using the parametric modeling file written by Patran's secondary development language. Matlab is integrated into the optimization procedure to conduct data processing for the result computed by Nastran. The needed parameters obtained are delivered to iSIGHT to conduct further analysis. And MIGA as well as SQP solver in iSIGHT's optimization toolbox are used to constitute optimization combining strategy to solve the problem. Meanwhile, we conduct dynamic characteristics analysis for launch vehicle's new strap-on configuration to test whether it satisfies the requirement of the attitude control design. The flowchart of the optimization for the new generation high-thrust strap-on launch vehicle's force transmission path based on iSIGHT is showed in Fig. 4.

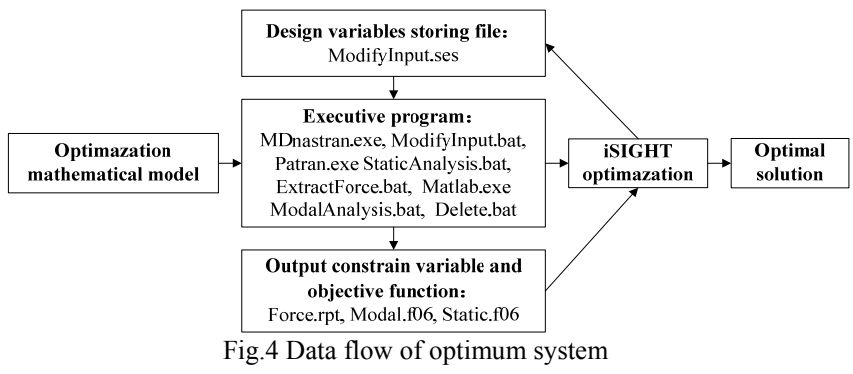

\section{THE OPTIMIZATION RESULT AND COMPARISON}

The optimization problem is solved by combinational optimization strategy, combining the global and local optimization algorithms. The optimization results of high-thrust launch vehicle's main linkage structure's load are showed in Fig. 5 and 6, the optimization results of auxiliary linkage structure's load is showed in Fig. 7. All results tend to be convergent.

Statically-determinate front strap-on configuration with the hyper-static strap-on configuration I after the optimization of force transmission path and this same configuration before the optimization in various external force conditions, their load-bearing structure's maximum loads are showed in table 3 . In this table, the variation range 1 represents the comparison of structural load between the configuration I after the optimization and the initial statically-determinate front strap-on configuration, the variation range 2 represents the comparison of structural load between the configuration I after the optimization and the initial configuration.

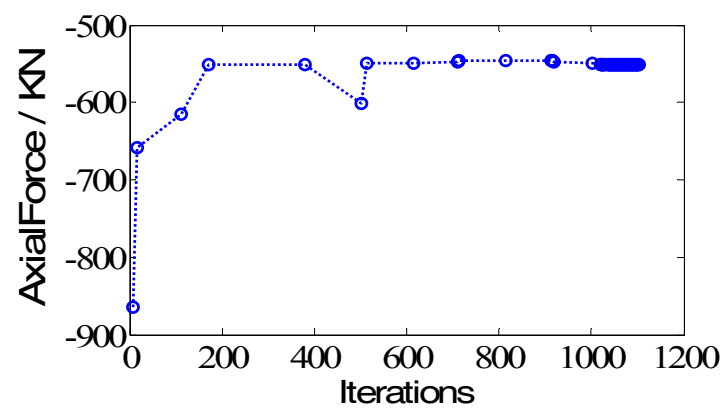

Fig.5 The optimization history of the main shaft's axial force

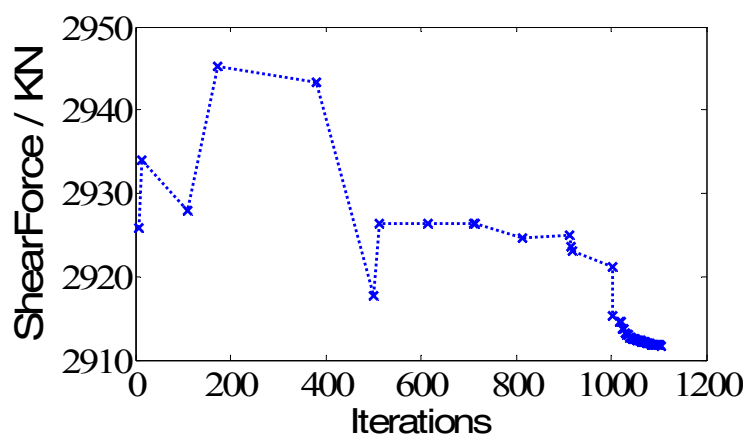

Fig.6 The optimization history of the main shaft's shear force

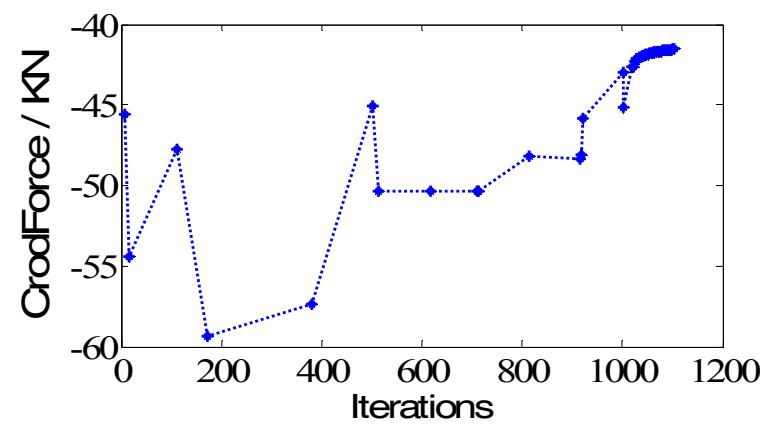

Fig.7 The optimization history of the auxiliary rod's axial force

TABLE 3 OPTIMIZATION RESULTS OF STRUCTURE MAXIMUM FORCE

\begin{tabular}{lccc}
\hline & $\begin{array}{c}\text { auxiliary } \\
\text { linkage rod's } \\
\text { axial force }\end{array}$ & $\begin{array}{c}\text { main linkage } \\
\text { shaft's } \\
\text { axial force }\end{array}$ & $\begin{array}{c}\text { main linkage } \\
\text { shaft's } \\
\text { shear force }\end{array}$ \\
\hline $\begin{array}{l}\text { initial configuration I } \\
{[\mathrm{KN}]}\end{array}$ & -52.2 & -828.4 & 2951.3 \\
$\begin{array}{l}\text { statically-determinate } \\
\text { front }\end{array}$ & 456.2 & -2123.3 & 4201.9 \\
strap-on configuration & & & \\
{$[\mathrm{KN}]$} & -41.5 & -551.9 & 2911.7 \\
$\begin{array}{l}\text { configuration I } \\
\text { optimization [KN] }\end{array}$ & -20.44 & -33.38 & -1.34 \\
variation range 1[\%] & -109.10 & -74.01 & -30.70 \\
variation range 2[\%] & & & \\
\hline
\end{tabular}


After the optimization for the configuration I's force transmission path, the main linkage shaft's axial force decreases by $33.38 \%$, the auxiliary linkage rod's axial force decreases by $20.44 \%$, the main linkage shaft's shear force decreases by $1.34 \%$. Compared with the statically-determinate front strap-on configuration, every kind of the force in the load-bearing structure decreases drastically, the structural force decreases by at least $30 \%$.

The natural frequencies of the high-thrust launch vehicle with the statically-determinate front strap-on configuration and configuration I before and after the optimization are showed in table 4 . In this table, frequency variation range 1 represents the comparison of the natural frequency between the configuration I after the optimization and the initial statically-determinate front strap-on configuration, frequency variation range 2 represents the comparison of the natural frequency between the configuration I after the optimization and before the optimization.

Compare with the statically-determinate front strap-on configuration and the configuration I, the dynamic property calculation results demonstrate the natural frequency has little variation after the optimization, although with small increase in the frequency's value. Meanwhile, the whole launch vehicle's fundamental frequency (indicated by $\mathbf{\text { ) }}$ ) is smaller than the booster's local first-order frequency (indicated by $\star$ ), which satisfies the requirement of the attitude control design.

TABLE 4 THE OPTIMIZATION RESULTS OF THE HIGH-THRUST LAUNCH VEHICLE'S NATURAL FREQUENCY

\begin{tabular}{|c|c|c|c|c|c|}
\hline order & $\begin{array}{l}\text { statically-determinate front } \\
\text { strap-on configuration }[\mathrm{Hz}]\end{array}$ & $\begin{array}{c}\text { configuration I } \\
\text { initial }[\mathrm{Hz}]\end{array}$ & $\begin{array}{c}\text { configuration I } \\
\text { optimization [Hz] }\end{array}$ & $\begin{array}{l}\text { variation } \\
\text { range } 1[\%]\end{array}$ & $\begin{array}{l}\text { variation } \\
\text { range } 2[\%]\end{array}$ \\
\hline 1 & 1.1150 & $1.097 \bigcirc$ & 1.1160 & 0.09 & 1.73 \\
\hline 2 & 1.115 & 1.097 & 1.116 & 0.09 & 1.73 \\
\hline 3 & $1.117 \star$ & $1.118 \star$ & $1.119 \star$ & 0.18 & 0.09 \\
\hline 4 & 1.150 & 1.178 & 1.194 & 3.83 & 1.36 \\
\hline 5 & 1.150 & 1.178 & 1.194 & 3.83 & 1.36 \\
\hline
\end{tabular}

\section{CONCLUSION}

This essay conducts FEM parametric modeling and analysis for the new generation high-thrust strap-on launch vehicle's preliminary plan. Based on the fact that this kind of launch vehicle's load design index is much greater than that of the current structure and the traditional statically-determinate strap-on configuration is more difficult to satisfy the high-thrust launch vehicle's design, we design one category of hyper-static strap-on linkage configurations. Through the further structural load analysis, an appropriate new strap-on configuration is selected. By integrating MSC.Patran/ Nastran and Matlab in iSIGHT, we use the combinational optimization strategy composed of MIGA and SQP to achieve the multi-objective optimization for the force transmission path.

Compared with the traditional statically-determinate strap-on linkage configuration, the force transmission path after the optimization obtained in this essay can decrease the linkage structure's fore by at least 30\%. Meanwhile, the dynamic characteristic analysis for the new strap-on configuration indicates that the new force transmission path satisfies the requirement of the attitude control design. The optimization design method demonstrated by this essay provides a reliable way for new strap-on launch vehicle's linkage equipment design.

\section{REFERENCES}

[1] He Wei, Liu Wei, Long Lehao, Heavy Launch Vehicle and Its Application, MISSILESAND SPACE VEHICLES. 1 (2011) 1-5.

[2] Feng Shaowei, Liu Zhusheng, Ma Zhonghui, Yao Shidong, Status of Application of Coupling Technology in Strap-on Launch Vehicle, MISSILESAND SPACE VEHICLES. 6 (2012) 20-23.

[3] Feng Shaowei, Research on the Mechanics Character of Hyper-static Strap-on Launch Vehicle, China Aerospace Science and Technology Corporation, 2013.

[4] Feng Shaowei, Liu Zhusheng, Luan Yu, Ma Zhonghui, Yao Shidong, Dynamic Analysis of Strap-on Equipment in Launch Vehicle Based on Different Tensile and Compressive Stiffness, MISSILESAND SPACE VEHICLES. 4 (2013) 9-13.

[5] Lu Yu, LONG Le-hao, et al, The world aerospace vehicle Book, second ed, Astronautics Press, 2007.

[6] Boggs P. T., Tolle J. W., Sequential quadratic programming for large-scale nonlinear optimization, Journal of Computational and Applied Mathematics. 124(1-2) (2000) 123-137.

[7] CHEN H., OOKA R., KATO S., Study on optimum design method for pleasant outdoor thermal environment using genetic algorithms and coupled simulation of convection, radiation and conduction, Building and Environment. 43(1) (2008) 18-31.

[8] HAN Bang-cheng, YUAN Qian, The Combinatorial Optimization Strategy for Large-Sized Magnetic Suspension Rotor in CMG System, Journal of Astronautics. 33(2) (2012) 275-280. 